\title{
One-Year Follow-Up of Healthy Older Adults with Electroencephalographic Risk for Neurocognitive Disorder After Neurofeedback Training
}

\author{
Graciela C. Alatorre-Cruz ${ }^{\mathrm{a}, \mathrm{b}}$, Thalía Fernández ${ }^{\mathrm{c}, *}$, Susana A. Castro-Chavira ${ }^{\mathrm{c}, \mathrm{d}}$, \\ Mauricio González-López ${ }^{\mathrm{c}}$, Sergio M. Sánchez-Moguel ${ }^{\mathrm{c}, \mathrm{e}}$ and Juan Silva-Pereyra ${ }^{\mathrm{a}}$ \\ ${ }^{a}$ Facultad de Estudios Superiores Iztacala, Universidad Nacional Autónoma de México, Estado de México, \\ México \\ ${ }^{\mathrm{b}}$ Department of Pediatrics. University of Arkansas for Medical Sciences, Little Rock, AR, USA \\ ${ }^{\mathrm{c}}$ Departamento de Neurobiología Conductual y Cognitiva, Instituto de Neurobiología, Universidad Nacional \\ Autónoma de México, Querétaro, México \\ ${ }^{\mathrm{d}}$ Institutt for Psykologi, Det Helsevitenskapelige Fakultet, Universitetet i Troms $\emptyset$ Norges Arktiske Universitet, \\ Tromsø, Tromsø, Norway \\ ${ }^{\mathrm{e}}$ Escuela Superior de Atotonilco de Tula, Universidad Autónoma del Estado de Hidalgo, Hidalgo, México
}

Accepted 1 December 2021

Pre-press 30 December 2021

\begin{abstract}
.
Background: In healthy older adults, excess theta activity is an electroencephalographic (EEG) predictor of cognitive impairment. In a previous study, neurofeedback (NFB) treatment reinforcing reductions theta activity resulted in EEG reorganization and cognitive improvement.

Objective: To explore the clinical applicability of this NFB treatment, the present study performed a 1-year follow-up to determine its lasting effects.

Methods: Twenty seniors with excessive theta activity in their EEG were randomly assigned to the experimental or control group. The experimental group received an auditory reward when the theta absolute power (AP) was reduced. The control group received the reward randomly.

Results: Both groups showed a significant decrease in theta activity at the training electrode. However, the EEG results showed that only the experimental group underwent global changes after treatment. These changes consisted of delta and theta decreases and beta increases. Although no changes were found in any group during the period between the posttreatment evaluation and follow-up, more pronounced theta decreases and beta increases were observed in the experimental group when the follow-up and pretreatment measures were compared. Executive functions showed a tendency to improve two months after treatment which became significant one year later.

Conclusion: These results suggest that the EEG and behavioral benefits of this NFB treatment persist for at least one year, which adds up to the available evidence contributing to identifying factors that increase its efficacy level. The relevance of this study lies in its prophylactic features of addressing a clinically healthy population with EEG risk of cognitive decline.
\end{abstract}

Keywords: Biofeedback, cognitive aging, EEG, follow-up, healthy aging, neurofeedback, older adults

*Correspondence to: Dra. Thalía Fernández, Laboratorio de Psicofisiología, Departamento de Neurobiología Conductual y Cognitiva, Instituto de Neurobiología, Universidad Nacional
Autónoma de México, Campus Juriquilla, Boulevard Juriquilla 3001, Querétaro 76230, México. Tel.: +52 4421926101 /Ext. 123, 124; E-mail: thaliafh@yahoo.com.mx. 


\section{INTRODUCTION}

Increases in life expectancy have resulted in a higher incidence of diseases related to aging $[1,2]$. In particular, old age is the primary risk factor for neurocognitive disorders (NCD) [3]; therefore, the number of patients with these disorders has increased. In accordance with the hypothesis that NCD may be a form of accelerated aging [4], declines in multiple cognitive processes, mainly in memory $[5,6]$ and executive functions $[7,8]$, are the most characteristic manifestations of cognitive decline. Moreover, the progression of such cognitive deficits becomes incapacitating in the advanced stages of NCD. Hence, the development of therapeutic methods aimed at slowing or preventing the progression of NCD has become increasingly relevant.

Measurement of the resting-state electroencephalogram (EEG) allows us to infer the baseline function of the brain [9], which implies that EEG may reflect age-related cognitive decline. EEG changes that occur with aging include a reduction in the voltage of the alpha rhythm and a slowing of the background alpha activity (posterior dominant rhythm), which results in a reduction of the alpha absolute power (AP) and the frequency of the alpha peak; however, Chang et al. [10] considered that this may be the effect of cerebral pathology (vascular or degenerative) that occurs with old age. Aging has also been related to a topographic reorganization of the alpha rhythm resulting in a spread to frontal areas [11]. This "frontalization" of alpha activity might be a consequence of age-related atrophy that occurs, especially in sensorimotor and prefrontal areas [10-14]. EEG in old age is also characterized by the appearance of scattered theta waves that are reflected in a diffuse increase in theta power and the occasional appearance of delta waves with temporal predominance [15-17]. In NCD, an exacerbation of the EEG features reported in healthy aging is observed, mainly, an increase in slow activity (delta and theta) [18-20] and a reduction in the posterior alpha rhythm [20-22]. Moreover, slow wave activity has been linked to the worst cognitive status during aging [23-26].

Prichep et al. [23] showed that these EEG features are not exclusive to patients with NCD. An excess of slow activity, mainly in the theta frequency range, is an excellent predictor of cognitive impairment, even 7-10 years before the disease is clinically apparent. Recently, Musaeus et al. [25] demonstrated the predictive power of excess theta in a sample of almost 400 older adults. In those with minor NCD, theta activity has been related to an increased risk of converting to major NCD [27-29].

Neurofeedback (NFB) is a neuroregulatory training tool that aims to modify specific characteristics of brain activity through the application of operant conditioning. In the particular case of EEG-based NFB, individuals can learn to modify their brain's electrical activity [30, 31]. This training has been used in older adults, both healthy and with NCD, to improve certain aspects of their cognitive performance. The rationale for developing an NFB protocol has varied among studies. Regarding major NCD, the purpose of NFB treatment has been to normalize abnormal EEG activity in patients. Surmeli et al. [32] and Luijmes et al. [33] used individualized NFB protocols for each patient based on their baseline EEG evaluations. Other studies investigated the effect of NFB on minor NCD with different protocols including the uptraining of SMR/theta [34], alpha [35], beta [36], and beta/alpha [37]. Even though the protocols were different, a common characteristic was the reinforcement of higher frequencies. Regarding healthy older adults, the rationale for protocol development has been of three types: 1) Lecomte et al. [38] aimed to induce an EEG pattern similar to that in expert meditators; 2) Wang \& Hsieh [39] tested a protocol that upregulated frontal midline theta activity and Reis et al. [40] increased alpha and theta based on what has been reported regarding task-induced EEG; and 3) another group of authors reinforced the production of higher-frequency activity based on the resting-state EEG characteristics associated with healthy aging (i.e., alpha [40, 41], SMR [42], beta [43, 44], and gamma [44]) or inhibited theta activity [45].

To date, there have been no studies that demonstrated whether the EEG activity during NFB was more similar to any one of these three conditions (meditation, task, or resting state). Our rationale for implementing NFB is in line with the third rationale based on the resting-state EEG characteristics observed in healthy aging.

Thus, our results from a previous study [45] led us to apply an NFB treatment that reinforced reductions in excessive theta activity, with the expectation of a concomitant cognitive improvement. Fourteen healthy adults over the age of 60 were randomly assigned to one of two groups: the experimental group, which received NFB training (consisting of a reward when absolute theta power was reduced), and the control group, which received sham training that consisted of a random delivery of the same auditory stimulus. Although both groups showed 
improvements in their EEG and behavioral measures, only the experimental group showed a significant tendency to normalize the EEG absolute power (AP), which consisted of a decrease in theta activity, mainly at the midline, and an increase in the alpha band in the left lateral electrodes. This was reflected in significantly higher scores in verbal processing.

Despite the recommendations that NFB studies incorporate a regular follow-up of the participants [46], there are only a couple of studies in older adults that address this concern. In both studies, Lavy et al. [35] and Marlats et al. [34] included individuals with minor NCD, and their follow-up periods were one month after treatment. In both studies, the NFB effect on cognition endured at the follow-up. Lavy et al. found that the improvement observed after only ten sessions of treatment was not sustained at the followup, in contrast to Marlats et al. [34] who observed that the EEG changes were sustained at the follow-up after 20 sessions of NFB. This might suggest that a larger number of sessions is required for the endurance of the changes produced by NFB.

The permanence of the effects attributable to NFB is crucial for it to be considered clinically useful. Thus, in the Becerra et al. study [45], we attempted to evaluate the effects of NFB two years after training; however, this was not possible: when the participants were contacted two years later, only those in the experimental group agreed to attend follow-up evaluations, whereas the control group participants refused to receive the NFB treatment.

Therefore, the main goal of the present study was to explore the permanence of the changes produced by NFB one year after completion of the training. Consistent with a previous study [45], we hypothesized that a decrease in the theta band, an increase in the alpha band, and an improvement in cognitive performance would occur after treatment and that, based on the few existing follow-up studies, these changes would persist a year later.

\section{MATERIALS AND METHODS}

\section{Participants}

Ninety-seven older adults were recruited and screened for inclusion criteria fulfillment from 2012 to 2018. Twenty right-handed healthy individuals older than 60 years of age (range $=60-81$ years) were selected for this study. The sample size calculation yielded a result of 9 subjects per group. This calculation for mean differences (one group) was performed using effect size data from the experimental group in our previous study [45]. The largest difference between the before and after measurements was used; thus, the statistical power was set at 13 , and the standardized difference was set at 11 , as described elsewhere [47]. Right-handedness was assessed with a Spanish version of the Edinburgh Handedness Inventory (LQ>+50) [48]. The participants had more than 9 years of schooling, scored more than 90 on the Spanish version for the Mexican population of the Wechsler adult intelligence scale-III (WAIS-III) [49] and received a normal global score on the short version of NEUROPSI, a brief neuropsychological test battery normalized in the Mexican population [50]. A specialist in geriatric psychiatry assessed all subjects to exclude participants with psychiatric or neurological disorders. The psychiatric examination included the Mini-Mental State Examination (MMSE) [51] and the global deterioration scale (GDS) [52]. Therefore, the behavioral assessment of cognition in all participants was normal (MMSE $\geq 27$; GDS $\leqq 2$ ). Moreover, the participants did not present signs of diabetes, anemia, hypercholesterolemia, or thyroid disease in clinical blood panel analyses, nor did they have uncontrolled hypertensive disease. The participants had an abnormally high theta AP value in at least one electrode of their EEG; this was an inclusion requirement because it has been shown that an excess theta AP is a predictor of cognitive decline seven years before clinical signs and symptoms appear [23]. A more detailed explanation is provided in the EEG recording and analysis section.

The participants provided written informed consent to participate in this study. This project (Ref: 030.H-RM) was approved by the Bioethics Committee of the Instituto de Neurobiología, Universidad Nacional Autónoma de México (UNAM), and it was performed in accordance with the Declaration of Helsinki and the Official Mexican Norms for experiments with human subjects.

These 20 subjects were assigned in a pseudorandom fashion (controlling for age, sex, and $\mathrm{z}$ theta $\mathrm{AP}$ value at the derivation in which this value was the highest) to one of two groups: the experimental group (EXP), which was given an NFB treatment that positively reinforced reductions in theta $\mathrm{AP}$, and the control group (CTL), which was administered a sham NFB treatment as described below. During treatment, two participants withdrew from the study.

Thus, the EXP group was composed of 10 participants (age (mean \pm standard deviation): $68.60 \pm 5.95$ 
Table 1

Demographic and cognitive characteristics of participants in the experimental and control groups before treatment

\begin{tabular}{|c|c|c|c|c|}
\hline & $\begin{array}{l}\text { Experimental } \\
\text { Percentage (n) }\end{array}$ & $\begin{array}{c}\text { Control } \\
\text { Percentage (n) }\end{array}$ & $\chi^{2}(1)$ & $p$ \\
\hline$\overline{\operatorname{Sex}}$ & & & 0.00 & 1.00 \\
\hline Male: & $50 \%(5)$ & $50 \%(4)$ & & \\
\hline Female & $50 \%(5)$ & $50 \%(4)$ & & \\
\hline GDS score & & & 2.88 & 0.22 \\
\hline 1 & $70 \%$ (7) & $100 \%(8)$ & & \\
\hline \multirow[t]{2}{*}{2} & $30 \%(3)$ & $0 \%$ & & \\
\hline & Mean (SD) & Mean (SD) & $\mathrm{t}(1,16)$ & $p$ \\
\hline Age & 67.50 (7.03), 60-81 y & $68.60(5.95), 61-77$ y & -0.36 & 0.72 \\
\hline Years of schooling & $15.00(4.57)$ & $15.40(3.47)$ & -0.21 & 0.83 \\
\hline MMSE & $28.50(.71)$ & $29.00(.94)$ & 1.14 & 0.25 \\
\hline NEUROPSI-total & $106.00(8.44)$ & $101.75(7.05)$ & 1.05 & 0.31 \\
\hline WAIS-IQ & $101.25(9.57)$ & $100.30(8.70)$ & 0.28 & 0.78 \\
\hline z AP theta & $2.75(1.03)$ & $3.60(1.51)$ & -1.35 & 0.19 \\
\hline
\end{tabular}

n, number of participants; SD, standard deviation; GDS, Global Deterioration Scale; MMSE, Mini-Mental State Examination; zAPG theta, z-values of theta absolute power with geometric power correction.

years, range: 60-81 years; years of schooling: $15.40 \pm 3.47 ; 5$ women), and the CTL group was composed of 8 participants (age: $67.50 \pm 7.03$ years, range: $61-77$ years; years of schooling: $15.00 \pm 4.57$; 4 women) since two of the subjects assigned to the CTL group withdrew from treatment before it concluded (see Table 1). There were no differences between the groups in age $(\mathrm{t}(16)=-0.36$, $p=0.72)$, sex $\left(\chi^{2}(1)=0.0, p=1.00\right)$, years of schooling $(\mathrm{t}(16)=-0.21, p=0.83)$, or cognitive status (MMSE: $\mathrm{t}(16)=1.14, p=0.25$; GDS: $\chi^{2}(1)=2.88$, $p=0.22$ ) before treatment. Additionally, there were no differences between the groups in the initial IQ scores from the WAIS-III (EXP: $100.30 \pm 8.70$; CTL: $101.25 \pm 9.57 ; \mathrm{t}(16)=0.28, p=0.78)$ or the global scores on the NEUROPSI (EXP: 101.75 \pm 7.05 ; CTL: $106 \pm 8.44 ; \mathrm{t}(16)=1.05, p=0.31)$.

The participants were evaluated using the WAISIII, NEUROPSI, and EEG at three time points: before (B), 2 months after (2mA), and 1 year after (1yA) NFB treatment.

\section{EEG recording and analysis}

The subjects were seated in a comfortable chair in a sound-proof, faradized, dimly-lit room. The referential EEG was recorded at rest with subjects' eyes closed for 15 min using a Medicid ${ }^{\mathrm{TM}}$ IV system and Track Walker ${ }^{\mathrm{TM}}$ v2.0 data system (Neuronic Mexicana, S.A.; Mexico). We used the appropriate cap (ElectroCap ${ }^{\mathrm{TM}}$, International Inc.; Eaton, Ohio) with 19 electrodes mounted and distributed according to the 10/20 international system, which were referenced to linked ear lobes. The EEG was digitized with a sampling rate of $200 \mathrm{~Hz}$ using a bandpass filter of $0.5-50 \mathrm{~Hz}$; the impedance was kept below $5 \mathrm{k} \Omega$.

The participants were not under the effects of any psychopharmacological medication and were instructed to not take any substance to induce sleep the night before recording. During the recording, they were instructed to keep their eyes closed and remain relaxed. The subjects were also cautioned not to fall asleep.

For the analysis, an expert electroencephalographer visually edited the EEG, selecting 24 artifactfree segments of $2.56 \mathrm{~s}$. The expert selected segments without intrinsic or extrinsic artifacts in any channel. The selected segments throughout the recording had to maintain the frequency and amplitude characteristics that the posterior rhythm had at the beginning of the recording to avoid activity associated with drowsiness. The individual QEEG analyses were performed using the fast Fourier transform to obtain cross-spectral matrices, and the AP with a geometric power correction [53] was calculated every $0.39 \mathrm{~Hz}$. With this correction, approximately $42 \%$ of the variance not related to physiological factors is reduced. The AP in each of the four classic frequency bands was obtained: delta $(1.5-3.5 \mathrm{~Hz})$, theta $(4-7.5 \mathrm{~Hz})$, alpha $(8-12.5 \mathrm{~Hz})$, and beta $(13-19.5 \mathrm{~Hz})$; these frequency ranges were the same as those used for the normative database [54] provided by MEDICID IV. Corresponding $\mathrm{z}$-values for AP were obtained by comparing each subject's values with the values from the normative database $[z=(\mathrm{x}-\overline{\mathrm{x}}) / \mathrm{S}$, where $\overline{\mathrm{x}}$ and $\mathrm{S}$ are the mean value and the standard deviation, respectively, of the normative sample]; a $\mathrm{z}$ value higher than 1.96 was considered abnormally high because it is 
approximately two standard deviations over the mean of the norms.

\section{Neurofeedback and sham treatments}

The corresponding treatment (NFB or sham) was applied using the EEG from the lead in which the most abnormally high zAP(theta) value was found. The participants in both groups were told that they would receive one of two available treatments without specifying that a sham treatment existed. They were also told that the training that would prove to be the most successful would be later offered to those who had not received it. No strategies to regulate their brain activity were given to the participants. NFB was conducted using a custom-made program adapted to TrackWalker software (see [45]). EEG was recorded from the electrode with the highest $\mathrm{z}[\mathrm{AP}$ theta], i.e., the target lead, referenced to the linked earlobes. The program analyzed theta AP in windows of $1,280 \mathrm{~ms}$, displacing $20 \mathrm{~ms}$ each time, and it emitted a $1,000 \mathrm{~Hz}$ tone (reward) when theta AP was below the selected threshold value. This threshold value was set in such a manner that the subject would receive the reward between $60 \%$ and $80 \%$ of the time following a continuous reinforcement schedule. Throughout the recording, the $\mathrm{z}$ value of the theta AP was computed and compared with the threshold. If this value was lower than the threshold, the reward was delivered. The participant was told that it was important to maintain the sound because it indicated that his or her brain was working well; consequently, the tone acquired a positive connotation. Each participant received 30 training sessions ( $30 \mathrm{~min}$ per session) over a period of ten to twelve weeks.

For every participant, a threshold was selected in the first session such that the participant received the reward approximately $70 \%$ of the time. The percentage of reward deliveries was quantified every three minutes: if the percentage was under $60 \%$, the threshold for the next 3-minute interval was increased, and if the percentage was over $80 \%$, the threshold was decreased. Across the training, no online artifact correction was implemented; however, the frequency that was trained is not within the range of frequencies most commonly affected by artifacts in EEG recordings, i.e., eye movements or electromyographic activity.

In the sham treatment, the conditions were the same as in the NFB treatment except that tone delivery and its duration were random, i.e., not contingent on the subject's EEG activity. Once the study was concluded, contingent NFB treatment was administered to the participants in the CTL group in accordance with the guidelines of the Declaration of Helsinki.

For all participants, plots showing their z[AP theta] values at the end of every session were available so they could check on their progress. The plots for the sham group showed simulated changes. These plots fulfilled a motivational purpose.

\section{Data analysis}

\section{EEG learning induced by NFB treatment}

As the first step, we evaluated whether the participants were able to learn to reduce the theta AP in the most abnormal electrode throughout NFB treatment, Thus, a Friedman test was conducted on data from both groups using the most abnormal z-value of theta AP per subject at $\mathrm{B}, 2 \mathrm{~mA}$, and $1 \mathrm{yA}$ to establish whether the treatment, in fact, induced the expected learning and whether this learning, if any, was sustained. Mann-Whitney tests were then performed on the differences between B and $2 \mathrm{~mA}$ as well as B and $1 \mathrm{yA}$ to establish if there was a difference in terms of the effect between groups, which would indicate us to what extent the effects were due to NFB-specific factors.

\section{EEG data}

Statistical analysis of the EEG data was performed using the statistical nonparametric mapping (SnPM) included in the standardized low-resolution brain electromagnetic tomography (sLORETA) software provided by the KEY Institute for Brain-Mind Research $[55,56]$. This method is a distribution-free test based on the estimation of the empirical probability distribution for the maximum t statistic [57]. The maximum $t$ and the extreme probability values as well as t- and $p$-values were reported. Then, corrections for multiple testing with a false discovery rate (FDR) were performed $(p<0.05)$.

The resting-state EEGs were recorded at three timepoints: B, $2 \mathrm{~mA}$, and $1 \mathrm{yA}$. The $\mathrm{z}$ scores of the $\mathrm{AP}$ in four frequency bands (i.e., delta, theta, alpha, and beta) were considered for these comparisons.

Each frequency band was separately analyzed as follows: 1) we compared the NFB effect on EEG AP in each group, separately, between pairs of time points (i.e., $2 \mathrm{~mA}$ versus $\mathrm{B}$; $1 \mathrm{yA}$ versus $2 \mathrm{~mA}$; 1yA versus $\mathrm{B}) ; 2$ ) in addition, we compared the groups with regard to the NFB effects, i.e., a) EXP[2mA-B] versus CTL[2mA-B], b) EXP[1yA-B] 

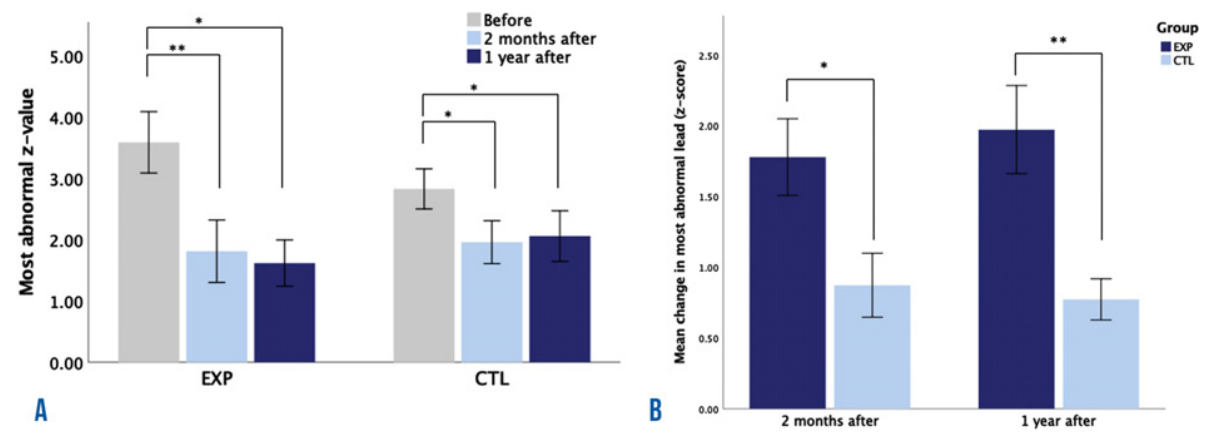

Fig. 1. Learning induced by neurofeedback treatment. A) The mean value of the most abnormal z-values of theta absolute power (with the geometric power correction) at three time points: before, 2 months after and 1 year after treatment, for the experimental group (EXP) and the control group (CTL). B) Differences between groups in the mean change of the most abnormal z-values of theta absolute power at 2 months after and 1 year after treatment. ${ }^{*} p<0.05$ and ${ }^{* *} p<0.01$ after correcting for pairwise comparisons.

versus CTL[1yA-B], and c) EXP[1yA-2mA] versus CTL[1yA-2mA]. $p$-values resulting from a set of comparisons were corrected by the FDR method (https://tools.carbocation.com/FDR).

\section{Cognitive assessment data}

Statistical analyses of the cognitive assessments (WAIS-III and NEUROPSI) were also performed with the SnPM method described above. FDR corrections were used to account for multiple comparisons $(p<0.05)$.

The WAIS-III and NEUROPSI tests were administered to the participants at B, $2 \mathrm{~mA}$ and $1 \mathrm{yA}$. The WAIS-III measures considered in the comparisons were the following: intelligence quotient (IQ), verbal comprehension index (VCI), working memory index (WMI), perceptual organization index (POI) and processing speed index (PSI), whereas the NEUROPSI measures were Orientation, Attention, Encoding, Language, Reading, Writing, and Executive functions: Conceptual and Motor, Memory recall and Total Score.

The WAIS-III and NEUROPSI tests were analyzed as follows: 1) we compared the NFB effect on the test scores in each group between pairs of time points, i.e., $2 \mathrm{~mA}$ versus $\mathrm{B}, 1 \mathrm{yA}$ versus $2 \mathrm{~mA}$, and $1 \mathrm{yA}$ versus $\mathrm{B} ; 2$ ) in addition, we compared the groups with regard to the NFB effects, i.e., a) EXP[2mA-B] versus CTL[2mA-B], b) EXP[1yA-B] versus CTL[1yA-B], and c) EXP[1yA-2mA] versus CTL[1yA-2mA]. $p$-values resulting from a set of comparisons were corrected by the FDR method (https://tools.carbocation.com/FDR).

Spearman correlation analyses were performed between the changes in NFB learning and changes in cognitive performance for every measure.

\section{RESULTS}

\section{Learning induced by NFB}

Figure 1 shows the mean and standard error of the most abnormal z-value per group at $\mathrm{B}, 2 \mathrm{~mA}$, and 1yA (Fig. 1A), as well as the comparison between groups for the effects at $2 \mathrm{~mA}$ and $1 \mathrm{yA}$ (Fig. 1B). We observed a significant effect of the treatment on the most abnormal individual z-value, which corresponded to the lead used during treatment, in both the EXP group $\left(\chi^{2}(2)=12.2, p=0.002\right)$ and the CTL group $\left(\chi^{2}(2)=9.25, p=0.01\right)$. Dunn-Bonferroni post $h o c$ tests were carried out, and significant differences in both groups were found between $B$ and $2 \mathrm{~mA}$ (EXP: $p=0.005$; CTRL: $p=0.018$ ) and between B and 1yA (EXP: $p=0.011$; CTRL: $p=0.037$ ), after the adjustment for pairwise comparisons. To establish whether the effect was different across groups, Mann-Whitney tests were conducted for the effect at $2 \mathrm{~mA}$ and $1 \mathrm{yA}$. The effect was larger in the experimental group at both $2 \mathrm{~mA}(U=16, p=0.034)$ and $1 \mathrm{yA}(U=9, p=0.004)$. Data of zAPtheta and the most abnormally higher lead for all participants at the three recording times is shown in Supplementary Table 1.

\section{EEG results}

\section{Delta zAP}

Comparisons between pairs of time points per group. As shown in Fig. 2, the EXP group had a lower delta zAP at 2mA than at B at the Fp1, Fp2, F4, C3, C4, P3, P4, O1, O2, T3, T4, T5, T6, Fz, Cz, and Pz locations $\left(\mathrm{t}_{\max }=2.85\right.$, global $\left.p=0.004\right)$, whereas the CTL group did not show significant differences in $\mathrm{zAP}\left(\mathrm{t}_{\max }=3.05\right.$, global $\left.p=0.4\right)$. No significant 


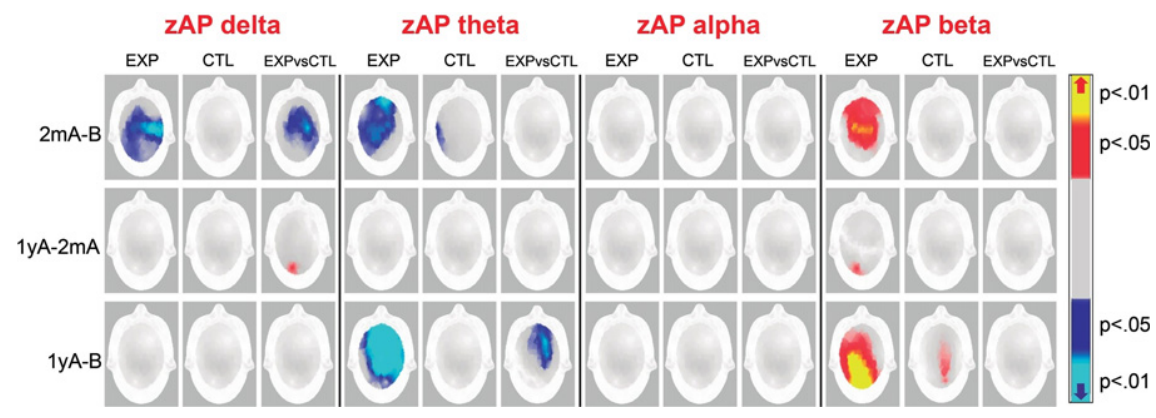

Fig. 2. Probability maps of zAP changes at 2 months after $(2 \mathrm{~mA})$ and 1 year after (1yA) neurofeedback treatment. There are four consecutive matrices, one for each frequency band (delta, theta, alpha, and beta). In each matrix, the first row represents the zAP changes that occurred from before (B) to $2 \mathrm{~mA}$ treatment, the second row from $2 \mathrm{~mA}$ to $1 \mathrm{yA}$ treatment, and the third row from B to 1yA treatment. The first and second columns of each matrix show changes in the experimental (EXP) and control (CTL) groups, respectively, and the third column shows the differences in the changes between groups. The color scale represents the probability of the differences between evaluations or groups; in red, the increases, and in blue, the decreases over time with respect to previous evaluations.

differences within groups were observed in the 1yA versus $2 \mathrm{~mA}$ or $1 \mathrm{yA}$ versus $\mathrm{B}$ comparisons.

Comparisons between groups with regard to NFB effects. There were significant differences between groups in the F3, F4, C3, C4, P3, P4, T6, Fz, and Cz locations $\left(\mathrm{t}_{\max }=2.9\right.$, global $\left.p=0.01\right)$. The decrease in delta zAP values at $2 \mathrm{~mA}$ than at B was greater in the EXP group than in the CTL group.

A significant difference between the groups was found in the $1 \mathrm{yA}-2 \mathrm{~mA}$ difference in the $\mathrm{O} 2$ site $\left(\mathrm{t}_{\max }=-3.0\right.$, global $\left.p=0.04\right)$; however, no significant within-group differences were found.

\section{Theta zAP}

Comparisons between pairs of time points per group. Figure 2 shows lower theta zAP values at $2 \mathrm{~mA}$ than at $\mathrm{B}$ in both groups; these differences were more widespread in the EXP group (Fp1, Fp2, F3, F4, C3, C4, P3, P4, F8, T3, T5, Fz, Cz, and Pz; $\mathrm{t}_{\max }=3.03$, global $p=0.002$ ) than in the CTL group (T3 and T5; $\mathrm{t}_{\max }=3.5$, global $p=0.02$ ).

The EXP group had a lower theta zAP at 1yA than at B in the Fp1, Fp2, F3, F4, C3, C4, P3, P4, $\mathrm{F} 8, \mathrm{~T} 4, \mathrm{~T} 6, \mathrm{Fz}, \mathrm{Cz}$, and $\mathrm{Pz}$ sites $\left(\mathrm{t}_{\max }=3.1\right.$, global $p=0.0002)$; meanwhile, the CTL group did not display differences in the $1 \mathrm{yA}$ versus $\mathrm{B}$ comparison $\left(\mathrm{t}_{\max }=3.7\right.$, global $\left.p=0.04\right)$. No differences in the theta zAP values were observed in the $1 \mathrm{yA}$ versus $2 \mathrm{~mA}$ comparisons.

Comparisons between groups with regard to NFB effects. Significant differences between groups were observed in the theta zAP in the 1yA-B difference; the EXP group displayed lower zAP values at 1yA than at $\mathrm{B}$ in the $\mathrm{Fp} 2, \mathrm{~F} 4, \mathrm{C} 4$, and $\mathrm{P} 4$ locations than the CTL group $\left(t_{\max }=3.10\right.$, global $\left.p=0.01\right)$.
No significant differences were found between groups in the $2 \mathrm{~mA}-\mathrm{B}$ and 1yA-2mA comparisons.

\section{Alpha zAP}

No significant differences were found within or between groups in any comparison (see Fig. 2).

\section{Beta zAP}

Comparisons between pairs of time points per group. As shown in Fig. 2, the EXP participants displayed a greater beta zAP at $2 \mathrm{~mA}$ than at $\mathrm{B}$ in the $\mathrm{Fp} 1$, Fp2, F3, F4, C3, C4, P3, P4, F7, Fz, Cz, and Pz sites $\left(\mathrm{t}_{\max }=-2.8\right.$, global $\left.p=0.007\right)$ than the CTL participants. The EXP group also displayed a greater beta zAP at $1 \mathrm{yA}$ than at $2 \mathrm{~mA}$ in the $\mathrm{O} 2$ site $\left(\mathrm{t}_{\max }=-3.5\right.$, global $p=0.05$ ). Both groups showed increased beta zAP values at $1 \mathrm{yA}$ than at $\mathrm{B}$, but this difference was more widespread in the EXP group ( $\mathrm{Fp} 2, \mathrm{~F} 4, \mathrm{C} 3, \mathrm{C} 4$, P3, O2, F7, F8, T4, T5, T6, Fz, Cz, and Pz; $t=-3.5$, $p=0.05)$ than in the CTL group $(\mathrm{C} 4, \mathrm{P} 4, \mathrm{O} 2$, and $\mathrm{Pz}$; $t=-3.7, p=0.04$ ).

The CTL group did not show differences in beta zAP values in the $2 \mathrm{~mA}-\mathrm{B}$ or the $1 \mathrm{yA}-2 \mathrm{~mA}$ differences.

Comparisons between groups with regard to the $N F B$ effects. No significant differences were found between groups in any comparison.

\section{Behavioral and cognitive results}

\section{Wais-III}

Comparisons between pairs of time points per group. No within-group differences were observed in the $2 \mathrm{~mA}$ versus $\mathrm{B}, 1 \mathrm{yA}$ versus $2 \mathrm{~mA}$, and $1 \mathrm{yA}$ versus $\mathrm{B}$ comparisons. See the statistical comparisons in Supplementary Table 2. 
Comparisons between groups with regard to NFB effects. As shown in Fig. 3, there were significant differences between groups concerning the NFB effects. For the global IQ, significant differences between groups were found regarding the changes between 2mA versus B (EXP: 3.400.4, CTL: -1.65 ; tmax $=2.79$, global $p=0.0451)$ and between $1 \mathrm{yA}$ versus 2mA (EXP: -3.90 , CTL: 2.38 ; $\operatorname{tmax}=-2.85$, $p=0.040$ ). Although significance was not reached, increased scores at $2 \mathrm{~mA}$ with respect to $B$ were found in the EXP group; conversely, these scores decreased in the CTL participants. On the other hand, significantly decreased scores at $1 \mathrm{yA}$ with respect to $2 \mathrm{~mA}$ were found in the EXP group, while the CTL participants showed increased scores. No significant differences were found in the $1 \mathrm{yA}$ versus $\mathrm{B}$ comparisons. See the statistical comparisons in Supplementary Table 3.

\section{Neuropsi}

Comparisons between pairs of time points per group. The EXP group displayed higher standardized scores in executive functions at $1 \mathrm{yA}$ than at $\mathrm{B}$ (B: $-0.5 \pm 0.99 ; 1 \mathrm{yA}: 0.11 \pm 0.75 ; \mathrm{t}_{\max }=2.86$, global $p<0.03$ ).

Meanwhile, the CTL group showed lower scores at $2 \mathrm{~mA}$ and $1 \mathrm{yA}$ than at $\mathrm{B}$ in executive functions (B: $0.07 \pm 0.55 ; 2 \mathrm{~mA}:-0.27 \pm 0.57$; $1 \mathrm{yA}:-0.12 \pm 0.60$; B versus $2 \mathrm{~mA}$ : $\mathrm{t}_{\max }=-2.97$, global $p<0.001$; B versus $1 \mathrm{yA}: \mathrm{t}_{\max }=-2.76$, global $\left.p<0.001\right)$. See the statistical comparisons in Supplementary Table 4.

Comparisons between groups with regard to NFB effects. Figure 4 shows the significant differences between the groups. The EXP group displayed an increase in executive functions at 1yA compared to $\mathrm{B}$, while the CTL group showed the inverse effect (EXP, B: $-0.5 \pm 0.99$; 1yA: $0.11 \pm 0.75$; CTL, B: $0.07 \pm 0.55$; $1 \mathrm{yA}:-0.12 \pm 0.60)$ resulting in differences between groups in executive functions at $1 \mathrm{yA}$ compared to B (EXP, mean difference, MD: 0.39; CLT, MD: $-0.19 ; \mathrm{t}_{\max }=2.91$, global $p<0.003$ ). See the statistical comparisons in Supplementary Table 5.

We found no significant correlations between the changes in NFB learning and changes in cognitive performance for every measure.

\section{DISCUSSION}

The aim of this study was to evaluate the effects of NFB training on cognition and resting-state EEG and to perform a 1-year follow-up to explore the permanence of the effects. A group of healthy older adults at risk of cognitive decline received NFB training that reinforced the reductions in theta $\mathrm{AP}$ at the most abnormal lead. This group was compared to a control group that received sham training. We first analyzed the changes in their theta z-values at the most abnormal lead to test whether they had learned to reduce abnormal theta activity in the target lead (Fig. 1). Both groups exhibited a decrease in their theta AP at the target lead; however, the effect was significantly larger in the EXP group, which suggested that NFB-specific learning was responsible for a part of this decrease in the EXP group. The remaining theta reduction found in both groups can be attributed to several nonspecific NFB factors. Changes observed in both training conditions could involve the use of metacognitive strategies that might have modified their EEG activity regardless of the contingency between EEG and reward delivery [58, 59]. Additionally, an expectancy [60] or placebo [61-63] effect could contribute to the reduction in theta in the target lead. Another factor that might have contributed to this theta reduction was the changes experienced by both groups in their lifestyles as a result of their involvement with the study. Since they were aware of the risk of developing future cognitive decline, they increased their activity level by introducing more reading, games such as Sudoku, music, dancing, exercise, etc. Moreover, their enrollment in this study demanded more interpersonal contact and involved performing additional activities such as driving or waking up earlier. The resting-state EEG recorded two months after training showed reductions in the delta and theta activity and an increase in beta in the EXP group, while in the CTL group, only a power reduction in the theta band in temporal leads was observed (Fig. 2, see 2mA- B).

In the 2 months versus 1 year after training (1yA $2 \mathrm{~mA}$ ) comparisons, only a significant increase in beta power in the left occipital lead was found in the EXP group. However, the observed decrease in theta and increase in beta were more pronounced when the EEG recorded one year after NFB was compared with the baseline EEG recorded before treatment in the EXP group.

In a previous study [45], a reduction in theta and an increase in beta activity were also observed. In that study, an increase in alpha was also observed; therefore, the mechanism of theta reduction was explained based on the work of Hughes et al. [64] showing that 

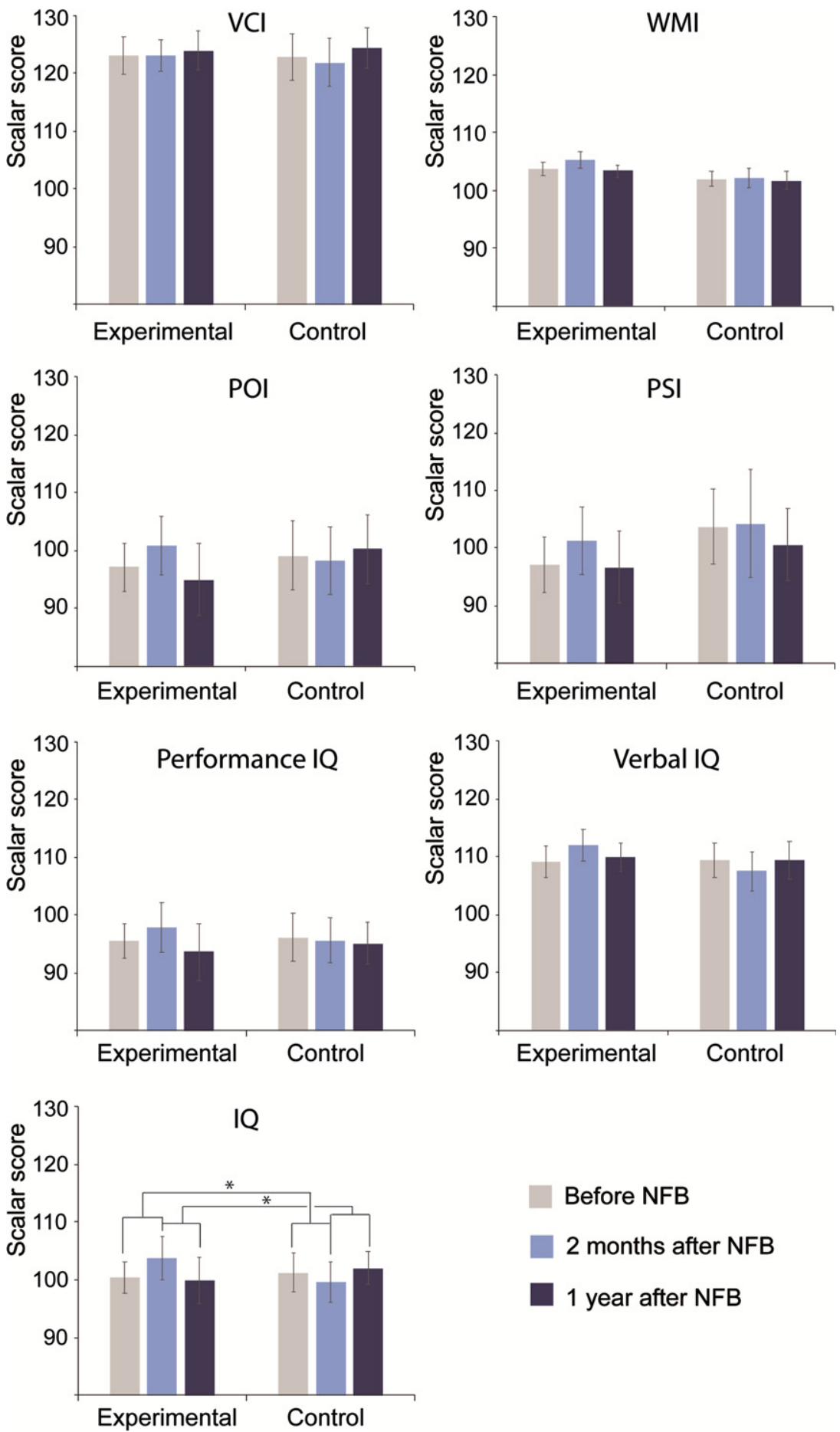

Before NFB

2 months after NFB

1 year after NFB

Fig. 3. Significant neuropsychological differences over time and between groups in the WAIS-III-R. The y-axes show the standardized scores resulting from neuropsychological assessments in both groups at the three evaluation time points: before treatment, two months after treatment and one year after treatment. VCI, verbal comprehension index; WMI, working memory index; POI, perceptual organization index; PSI, processing speed index; IQ, intelligence quotient. ${ }^{*} p<0.05,{ }^{* *} p<0.01$. 

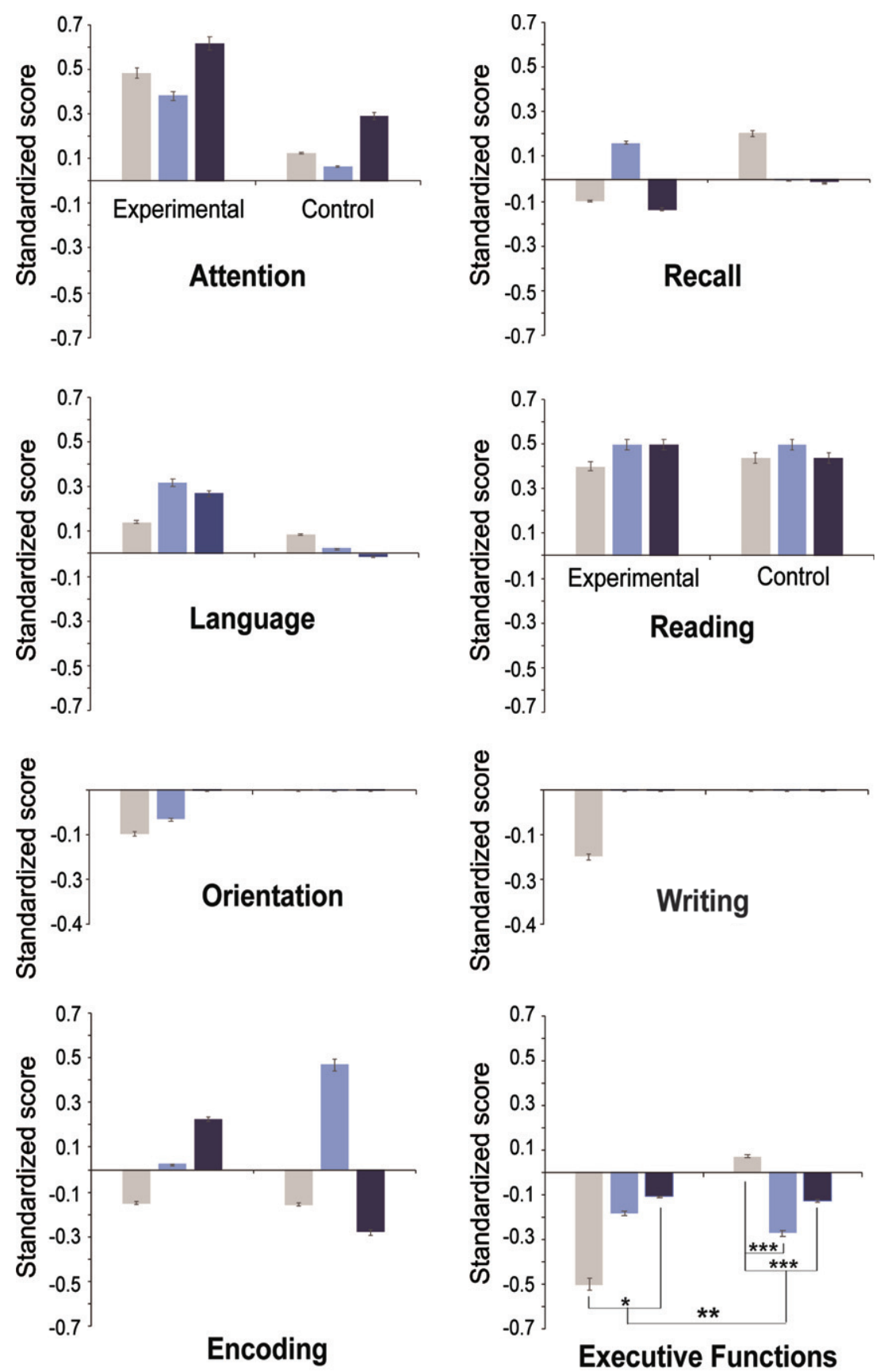

Before NFB $\quad 2$ months after NFB $\square 1$ year after NFB

Fig. 4. Significant neuropsychological differences over time and between groups in the NEUROPSI. The y-axes show the standardized scores resulting from neuropsychological assessments in both groups at the three evaluation time points: before treatment, two months after treatment and one year after treatment. ${ }^{*} p<0.05,{ }^{* *} p<0.01$.

alpha or theta production depends on the depolarization of the same thalamic neurons. In the present study, such an alpha increase was not observed, but an increase in beta activity emerged. Schutte et al.
[65] proposed that a beta increase may represent a quenching signal toward the subcortical structures that directs cortical theta activity and may downregulate reward anticipation in laboratory conditions. This 
mechanism may explain our results. The increase in beta and decrease in theta during resting-state EEG have been associated with increased adaptability during a reversal learning task $[66,67]$. This means that to accurately perform this task, cognitive flexibility for an optimal trade-off between exploiting the acquired knowledge and exploring alternative response options is required to make the best decision [68]. This agrees with the neuropsychological results obtained in the executive function domain, which tended to improve at two months after training and reached significance one year later.

In the EXP group, NFB treatment induced an increase in the overall EEG frequency. This finding is in agreement with our previous study [45], in which theta AP decreased and alpha and beta AP increased. As we hypothesized, the changes produced by the NFB treatment in the theta and beta frequency bands not only persisted but were also enhanced one year later. It has been proposed that the changes produced by NFB comprise a reorganization of the overall EEG activity and do not merely imply local changes associated with the target electrode and training frequency $[30,69,70]$.

In addition, the present study showed a decrease in delta activity after treatment, which was not observed in the previous study; this may have been because in the present study a delta excess in $80 \%$ of the participants in at least one lead before training was observed; while in Becerra's study, only two out of seven (28\%) participants showed a delta excess. After training, delta underwent reductions that may have been associated with an EEG reorganization produced by NFB and may mainly have affected the abnormal activity guiding it toward normality. On the other hand, alpha activity was not modified.

In older adults, an inverse relationship between EEG frequency and cognitive decline has been observed, i.e., higher frequency EEGs correspond to individuals with better cognitive status [19, 71-73]. This finding agrees with results associating restingstate EEG activity with cognition in healthy older adults [26].

In terms of the neuropsychological assessments, both groups differed in terms of performance at $2 \mathrm{~mA}$ and 1yA, compared to their baseline evaluation. The EXP group showed an improvement in their total IQ, which was not evident in the subjects' performance both $2 \mathrm{~mA}$ and 1yA treatment; however, in the CTL group, worse performance was observed 1yA sham training, even though some improvements were observed $2 \mathrm{~mA}$ treatment. An exception was the attention process, which tended to improve 1yA training regardless of the treatment received (Fig. 3).

According to the consensus on the reporting and experimental design of clinical and cognitivebehavioral neurofeedback studies (CRED-nf) checklist [46], all the essential criteria were satisfied except for artifact correction during the training sessions (criterion 3d), and plotting within-session and between-session regulation blocks of feedback variable(s), as well as pre-to-post resting baselines or contrasts (criterion 5b). Criterion $2 \mathrm{~b}$ was partially satisfied because participants were blind to the treatment, but experimenters were not blind to the conditions. It is necessary to consider that this checklist was published after this study was performed.

The essential limitation of this study was the small sample size; therefore, further research with larger sample sizes should be conducted. Additionally, studies using other neuroimaging methods, such as fMRI and ERPs, could provide insight into the functional changes produced by this NFB training that underlie the observed cognitive changes.

Since the participants of this study were healthy older adults, it might be considered that this NFB training was used with the purpose of enhancing performance; however, this was not the case because, even though our subjects exhibited normal cognitive performance (measured with behavioral methods, i.e., neuropsychological batteries) and systemic levels, they had excess theta EEG activity, which represents a risk for cognitive decline [23]. More than only a risk factor, this feature might involve compensatory cognitive strategies to reach the expected performance level, which has been demonstrated in previous studies from our group using event-related potentials $[74,75]$ and suggests that, in these individuals, there is a preclinical cognitive decline occurring that is not yet behaviorally expressed. Thus, our treatment is of a prophylactic nature.

As mentioned before, several rationales have been considered for the development of NFB protocols that address enhancements in cognitive performance in healthy elderly individuals. It is important to address this issue because one such rationale seems to be opposite to ours, i.e., the increase in frontal midline theta activity (fmTheta) [76, 77]. We consider that there are some problems with such a rationale and the evidence that has been presented. First, it is known that resting-state EEG activity differs from task-induced EEG activity, where a synchronization of theta activity and a desynchronization of alpha activity are often observed [78]. However, for an 
increase in fmTheta activity during NFB treatment to make sense, it would be logical to reinforce it during a task where it is expected to increase; however, this has not been the case. For example, Wang and Hsieh [39] reported changes associated with resting-state EEG using a task-induced EEG rationale. Second, the effectiveness of NFB protocols is often considered with respect to studies that include very few sessions. Such is the case of a study that aimed to uptrain fmTheta activity conducted by Reis et al. [40], which consisted of 8 sessions in total and only 4 sessions of theta uptraining. Considering the aforementioned arguments, it is difficult to establish whether the effects shown in such studies are actually due to NFB-specific factors (i.e., the operant conditioning of fmTheta upregulation) or if they reflect other nonspecific components.

A strength of our study is that 30 sessions of treatment were applied. As previously mentioned, conclusions about the effectiveness of NFB treatment are often drawn from studies that have only included a few sessions, and there are even reports that a single session of NFB produced the expected learning $[70,79]$. We are very surprised by these assertions because even in taste aversive learning [80, 81], which constitutes learning for survival, individuals are infrequently able to learn in a single session.

On the other hand, the results of the study by Becerra et al. [45] were replicated by the same laboratory, and this is one step toward the improvement of the available evidence regarding the efficacy of this NFB treatment [82] in this population. Moreover, this is the first NFB study in older adults with a followup of one year. Our results are also relevant because of the focus on the early detection of risks for cognitive decline and on the development of a prophylactic intervention, whose effects have been shown to be sustained at least one year after treatment.

\section{ACKNOWLEDGMENTS}

The authors acknowledge Leonor Casanova, Lourdes Lara, Teresa Álvarez and Bertha Esquivel for administrative support and Héctor Belmont, Saulo Hernández, Monserrat Palacios and Ricardo Bravo for technical assistance. We also thank Dr. Lourdes Díaz-Comas for the design of the software used to administer the treatments. This research was supported by project IN200817 from PAPIIT- DGAPA, Universidad Nacional Autónoma de México.

Authors' disclosures available online (https:// www.j-alz.com/manuscript-disclosures/21-5538).

\section{SUPPLEMENTARY MATERIAL}

The supplementary material is available in the electronic version of this article: https://dx.doi.org/ 10.3233/JAD-215538.

\section{REFERENCES}

[1] World Health Organization (2015) World report on ageing and health. World Health Organization. https://apps.who. int/iris/handle/10665/186463.

[2] World Health Organization (2021) Ageing and Health, https://www.who.int/news-room/fact-sheets/detail/ageingand-health.

[3] American Psychiatric Association (2013) Diagnostic and Statistical Manual of Mental Disorders, American Psychiatric Association, Arlington, VA.

[4] Fjell AM, Walhovd KB (2010) Structural brain changes in aging: Courses, causes and cognitive consequences. Rev Neurosci 21, 187-221.

[5] Borella E, Carretti B, de Beni R (2008) Working memory and inhibition across the adult life-span. Acta Psychol 128, 33-44.

[6] Rhodes RE, Katz B (2017) Working memory plasticity and aging. Psychol Aging 32, 51-59.

[7] Mani TM, Bedwell JS, Miller LS (2005) Age-related decrements in performance on a brief continuous performance test. Arch Clin Neuropsychol 20, 575-586.

[8] Verhaeghen P, Cerella J (2012) Everything we know about aging and response times: A meta-analytic integration. In Handbook of Cognitive Aging: Interdisciplinary Perspectives, SAGE Publications, Inc., pp. 134-150.

[9] Baars BJ, Gage NM (2010) Mind and brain. In Cognition, Brain, and Consciousness. Elsevier, pp. 2-31.

[10] Chang BS, Schomer DL, Niedermeyer E (2012) Normal EEG and sleep: Adults and elderly. Niedermeyer's Electroencephalography: Basic Principles, Clinical Applications, and Related Fields, pp. 183-214.

[11] Gasser T, Bächer P, Möcks J (1982) Transformations towards the normal distribution of broad band spectral parameters of the EEG. Electroencephalogr Clin Neurophysiol 53, 119-124.

[12] Knyazeva MG, Barzegaran E, Vildavski VY, Demonet JF (2018) Aging of human alpha rhythm. Neurobiol Aging 69, 261-273.

[13] Smailovic U, Jelic V (2019) Neurophysiological markers of Alzheimer's disease: Quantitative EEG approach. Neurol Ther 8, 37-55.

[14] Bakkour A, Morris JC, Wolk DA, Dickerson BC (2013) The effects of aging and Alzheimer's disease on cerebral cortical anatomy: Specificity and differential relationships with cognition. Neuroimage 76, 332-344.

[15] Aird RB, Gastaut Y (1959) Occipital and posterior electroencephalographic ryhthms. Electroencephalogr Clin Neurophysiol 11, 637-656.

[16] Busse EW, Barnes RH, Friedman EL, Kelty EJ (1956) Psychological functioning of aged individuals with normal and abnormal electroencephalograms: I. A study of nonhospitalized community volunteers. J Nerv Mental Dis 124, 135-141.

[17] Gil-Nagel A, Parra J, Irirarte J, Kanner A (2002) Manual de electroencefalografía, Mc Graw Hill Interamericana, Madrid. 
[18] Moretti D v., Babiloni C, Binetti G, Cassetta E, Dal Forno G, Ferreric F, Ferri R, Lanuzza B, Miniussi C, Nobili F, Rodriguez G, Salinari S, Rossini PM (2004) Individual analysis of EEG frequency and band power in mild Alzheimer's disease. Clin Neurophysiol 115, 299-308.

[19] Prichep LS, John ER, Ferris SH, Reisberg B, Almas M, Alper K, Cancro R (1994) Quantitative EEG correlates of cognitive deterioration in the elderly. Neurobiol Aging 15, 85-90.

[20] van der Hiele K, Vein AA, Reijntjes RHAM, Westendorp RGJ, Bollen ELEM, van Buchem MA, van Dijk JG, Middelkoop HAM (2007) EEG correlates in the spectrum of cognitive decline. Clin Neurophysiol 118, 1931-1939.

[21] van der Hiele K, Bollen ELEM, Vein AA, Reijntjes RHAM, Westendorp RGJ, van Buchem MA, Middelkoop HAM, van Dijk JG (2008) EEG markers of future cognitive performance in the elderly. J Clin Neurophysiol 25, 83-89.

[22] Smailovic U, Koenig T, Kåreholt I, Andersson T, Kramberger MG, Winblad B, Jelic V (2018) Quantitative EEG power and synchronization correlate with Alzheimer's disease CSF biomarkers. Neurobiol Aging 63, 88-95.

[23] Prichep LS, John ER, Ferris SH, Rausch L, Fang Z, Cancro R, Torossian C, Reisberg B (2006) Prediction of longitudinal cognitive decline in normal elderly with subjective complaints using electrophysiological imaging. Neurobiol Aging 27, 471-481.

[24] Prichep LS (2007) Quantitative EEG and electromagnetic brain imaging in aging and in the evolution of dementia. Ann N Y Acad Sci 1097, 156-167.

[25] Musaeus CS, Engedal K, Høgh P, Jelic V, Mørup M, Naik M, Oeksengaard AR, Snaedal J, Wahlund LO, Waldemar G, Andersen BB (2018) EEG theta power is an early marker of cognitive decline in dementia due to Alzheimer's disease. $J$ Alzheimers Dis 64, 1359-1371.

[26] Roca-Stappung M, Fernández T, Becerra J, MendozaMontoya O, Espino M, Harmony T (2012) Healthy aging: Relationship between quantitative electroencephalogram and cognition. Neurosci Lett 510, 115-120.

[27] Rossini PM, del Percio C, Pasqualetti P, Cassetta E, Binetti G, Dal Forno G, Ferreri F, Frisoni G, Chiovenda P, Miniussi C, Parisi L, Tombini M, Vecchio F, Babiloni C (2006) Conversion from mild cognitive impairment to Alzheimer's disease is predicted by sources and coherence of brain electroencephalography rhythms. Neuroscience 143, 793-803.

[28] Moretti DV, Pievani M, Fracassi C, Binetti G, Rosini S, Geroldi C, Zanetti O, Rossini PM, Frisoni GB (2009) Increase of theta/Gamma and Alpha3/Alpha2 ratio is associated with amygdalo-hippocampal complex atrophy. $J$ Alzheimers Dis 17, 349-357.

[29] Moretti DV, Frisoni GB, Fracassi C, Pievani M, Geroldi C, Binetti G, Rossini PM, Zanetti O (2011) MCI patients' EEGs show group differences between those who progress and those who do not progress to AD. Neurobiol Aging 32, 563-571.

[30] Dessy E, Mairesse O, van Puyvelde M, Cortoos A, Neyt X, Pattyn N (2020) Train your brain? Can we really selectively train specific EEG frequencies with neurofeedback training. Front Hum Neurosci 14, 22.

[31] Egner T, Sterman MB (2006) Neurofeedback treatment of epilepsy: From basic rationale to practical application. Expert Rev Neurotherap 6, 247-257.

[32] Surmeli T, Eralp E, Mustafazade I, Kos H, Ozer GE, Surmeli OH (2015) Quantitative EEG neurometric analysisguided neurofeedback treatment in dementia: 20 cases. How neurometric analysis is important for the treatment of dementia and as a biomarker? Clin EEG Neurosci 47, 118-133.

[33] Luijmes RE, Pouwels S, Boonman J (2016) L'efficacité du neurofeedback sur le fonctionnement cognitif chez les patients atteints de la maladie d'Alzheimer : Résultats préliminaires. Neurophysiol Clin 46, 179-187.

[34] Marlats F, Bao G, Chevallier S, Boubaya M, DjabelkhirJemmi L, Wu YH, Lenoir H, Rigaud AS, Azabou E (2020) SMR/theta neurofeedback training improves cognitive performance and EEG activity in elderly with mild cognitive impairment: A pilot study. Front Aging Neurosci $12,147$.

[35] Lavy Y, Dwolatzky T, Kaplan Z, Guez J, Todder D (2019) Neurofeedback improves memory and peak alpha frequency in individuals with mild cognitive impairment. Appl Psychophysiol Biofeedback 44, 41-49.

[36] Jang JH, Kim J, Park G, Kim H, Jung ES, Cha JY, Kim CY, Kim S, Lee JH, Yoo H (2019) Beta wave enhancement neurofeedback improves cognitive functions in patients with mild cognitive impairment: A preliminary pilot study. Medicine (Baltimore) 98, e18357.

[37] Jirayucharoensak S, Israsena P, Pan-Ngum S, Hemrungrojn S, Maes M (2019) A game-based neurofeedback training system to enhance cognitive performance in healthy elderly subjects and in patients with amnestic mild cognitive impairment. Clin Interven Aging 14, 347-360.

[38] Lecomte G, Juhel J (2011) The effects of neurofeedback training on memory performance in elderly subjects. Psychology 2, 846-852.

[39] Wang J-R, Hsieh S (2013) Neurofeedback training improves attention and working memory performance. Clin Neurophysiol 124, 2406-2420.

[40] Reis J, Portugal AM, Fernandes L, Afonso N, Pereira M, Sousa N, Dias NS (2016) An alpha and theta intensive and short neurofeedback protocol for healthy aging workingmemory training. Front Aging Neurosci 8, 157.

[41] Angelakis E, Stathopoulou S, Frymiare JL, Green DL, Lubar JF, Kounios J (2007) EEG neurofeedback: A brief overview and an example of peak alpha frequency training for cognitive enhancement in the elderly. Clin Neuropsychol 21, 110-129.

[42] Campos da Paz VK, Garcia A, Campos da Paz Neto A, Tomaz C (2018) SMR neurofeedback training facilitates working memory performance in healthy older adults: A behavioral and EEG study. Front Behav Neurosci 12, 321.

[43] Gomez-Pilar J, Corralejo R, Nicolas-Alonso LF, Álvarez D, Hornero R (2016) Neurofeedback training with a motor imagery-based BCI: Neurocognitive improvements and EEG changes in the elderly. Med Biol Eng Comput 54, 1655-1666.

[44] Staufenbiel SM, Brouwer AM, Keizer AW, van Wouwe NC (2014) Effect of beta and gamma neurofeedback on memory and intelligence in the elderly. Biol Psychol 95, 74-85.

[45] Becerra J, Fernández T, Roca-Stappung M, Díaz-Comas L, Galán L, Bosch J, Espino M, Moreno AJ, Harmony T (2012) Neurofeedback in healthy elderly human subjects with electroencephalographic risk for cognitive disorder. $J$ Alzheimers Dis 28, 357-367.

[46] Ros T, Enriquez-Geppert S, Zotev V, Young KD, Wood G, Whitfield-Gabrieli S, Wan F, Vuilleumier P, Vialatte F, van de Ville D, Todder D, Surmeli T, Sulzer JS, Strehl U, Sterman MB, Steiner NJ, Sorger B, Soekadar SR, Sitaram R, Sherlin LH, Schönenberg M, Scharnowski F, Schabus M, Rubia K, Rosa A, Reiner M, Pineda JA, Paret C, Ossadtchi 
A, Nicholson AA, Nan W, Minguez J, Micoulaud-Franchi JA, Mehler DMA, Lührs M, Lubar J, Lotte F, Linden DEJ, Lewis-Peacock JA, Lebedev MA, Lanius RA, Kübler A, Kranczioch C, Koush Y, Konicar L, Kohl SH, Kober SE, Klados MA, Jeunet C, Janssen TWP, Huster RJ, Hoedlmoser K, Hirshberg LM, Heunis S, Hendler T, Hampson M, Guggisberg AG, Guggenberger R, Gruzelier JH, Göbel RW, Gninenko N, Gharabaghi A, Frewen P, Fovet T, Fernández T, Escolano C, Ehlis AC, Drechsler R, Christopher Decharms R, Debener S, de Ridder D, Davelaar EJ, Congedo M, Cavazza M, Breteler MHM, Brandeis D, Bodurka J, Birbaumer N, Bazanova OM, Barth B, Bamidis PD, Auer T, Arns M, Thibault RT (2020) Consensus on the reporting and experimental design of clinical and cognitive-behavioural neurofeedback studies (CRED-nf checklist). Brain 143, 1674-1685.

[47] García-García JA, Reding-Bernal A, López-Alvarenga JC (2013) Cálculo del tamaño de la muestra en investigación en educación médica. Invest Educ Méd 2, 217-224.

[48] Oldfield RC (1971) The assessment and analysis of handedness: The Edinburgh inventory. Neuropsychologia 9, 97-113.

[49] Weschler D (2003) Escala Wechsler de Inteligencia para Adultos III, Manual técnico., Manual Moderno, México.

[50] Ostrosky-Solís F, Ardila A, Rosselli M (1999) NEUROPSI: A brief neuropsychological test battery in Spanish with norms by age and educational level. J Int Neuropsychol Soc 5, 413-433.

[51] Petersen RC, Smith GE, Waring SC, Ivnik RJ, Tangalos EG, Kokmen E (1999) Mild cognitive impairment: Clinical characterization and outcome. Arch Neurol 56, 303-308.

[52] Reisberg B, Ferris SH, de Leon MJ, Crook T (1982) The global deterioration scale for assessment of primary degenerative dementia. Am J Psychiatry 139, 1136-1139.

[53] Hernández JL, Valdés P, Biscay R, Virues T, Szava S, Bosch J, Riquenes A, Clark I (1994) A global scale factor in brain topography. Int J Neurosci 76, 267-278.

[54] Valdés P, Biscay R, Galán L, Bosch J, Zsava S, Virués T (1990) High resolution spectral EEG norms topography. Brain Topogr 3, 281-82.

[55] Pascual-Marqui RD (2001) Low resolution brain electromagnetic tomography (LORETA): The technique, its validation, and methods of analysis. J Neurother 4, 31-33.

[56] Pascual-Marqui RD (2007) Discrete, 3D distributed, linear imaging methods of electric neuronal activity. Part 1: Exact, zero error localization. arXiv:0710.3341

[57] Nichols TE, Holmes AP (2002) Nonparametric permutation tests for functional neuroimaging: A primer with examples. Hum Brain Mapp 15, 1-25.

[58] Huang C, Zhang H, Huang J, Duan C, Kim JJ, Ferrari M, Hu CS (2020) Stronger resting-state neural oscillations associated with wiser advising from the 2 nd- but not the 3 rd-person perspective. Sci Rep 10, 12677.

[59] Knowles MM, Wells A (2018) Single dose of the Attention Training Technique increases resting alpha and betaoscillations in frontoparietal brain networks: A randomized controlled comparison. Front Psychol 9, 1768.

[60] Schönenberg M, Weingärtner AL, Weimer K, Scheeff J (2021) Believing is achieving - On the role of treatment expectation in neurofeedback applications. Prog Neuropsychopharmacol Biol Psychiatry 105, 110129.

[61] Huneke NTM, Brown CA, Burford E, Watson A, TrujilloBarreto NJ, El-Deredy W, Jones AKP (2013) Experimental placebo analgesia changes resting-state alpha oscillations. PLoS One 8, 78278.
[62] Leuchter AF, Cook IA, Witte EA, Morgan M, Abrams M (2002) Changes in brain function of depressed subjects during treatment with placebo. Am J Psychiatry 159, 122-129.

[63] Li L, Wang H, Ke X, Liu X, Yuan Y, Zhang D, Xiong D, Qiu Y (2016) Placebo analgesia changes alpha oscillations induced by tonic muscle pain: EEG frequency analysis including data during pain evaluation. Front Comput Neurosci $10,45$.

[64] Hughes SW, Crunelli V (2007) Just a phase they're going through: The complex interaction of intrinsic high-threshold bursting and gap junctions in the generation of thalamic $\alpha$ and $\theta$ rhythms. Int $J$ Psychophysiol 64, 3-17.

[65] Schutte I, Kenemans JL, Schutter DJLG (2017) Restingstate theta/beta EEG ratio is associated with reward- and punishment-related reversal learning. Cogn Affect Behav Neurosci 17, 754-763.

[66] Schutter DJLG, van Honk J (2005) Electrophysiological ratio markers for the balance between reward and punishment. Cogn Brain Res 24, 685-690.

[67] Massar SAA, Kenemans JL, Schutter DJLG (2014) Restingstate EEG theta activity and risk learning: Sensitivity to reward or punishment? Int J Psychophysiol 91, 172-177.

[68] Daw ND, O'Doherty JP, Dayan P, Seymour B, Dolan RJ (2006) Cortical substrates for exploratory decisions in humans. Nature 441, 876-879.

[69] Fernández T, Harmony T, Fernández-Bouzas A, DíazComas L, Prado-Alcalá RA, Valdés-Sosa P, Otero G, Bosch J, Galán L, Santiago-Rodríguez E, Aubert E, García-Martínez F (2007) Changes in EEG current sources induced by neurofeedback in learning disabled children. An exploratory study. Appl Psychophysiol Biofeedback 32, 169-183.

[70] Kober SE, Witte M, Neuper C, Wood G (2017) Specific or nonspecific? Evaluation of band, baseline, and cognitive specificity of sensorimotor rhythm- and gamma-based neurofeedback. Int J Psychophysiol 120, 1-13.

[71] Fernández A, Hornero R, Mayo A, Poza J, Gil-Gregorio P, Ortiz T (2006) MEG spectral profile in Alzheimer's disease and mild cognitive impairment. Clin Neurophysiol 117, 306-314.

[72] Fernández A, Turrero A, Zuluaga P, Gil-Gregorio P, del Pozo F, Maestu F, Moratti S (2013) MEG delta mapping along the healthy aging-alzheimer's disease continuum: Diagnostic implications. J Alzheimers Dis 35, 495-507.

[73] Stomrud E, Hansson O, Zetterberg H, Blennow K, Minthon L, Londos E (2010) Correlation of longitudinal cerebrospinal fluid biomarkers with cognitive decline in healthy older adults. Arch Neurol 67, 217-223.

[74] Alatorre-Cruz GC, Silva-Pereyra J, Fernández T, Rodríguez-Camacho MA (2019) Poor working memory performance in healthy elderly adults with electroencephalographic risk of cognitive decline affects syntactic processing. Clin Neurophysiol 130, 2222-2230.

[75] Sánchez-Moguel SM, Alatorre-Cruz GC, Silva-Pereyra J, González-Salinas S, Sanchez-Lopez J, Otero-Ojeda GA, Fernández T (2018) Two different populations within the healthy elderly: Lack of conflict detection in those at risk of cognitive decline. Front Hum Neurosci 11, 658.

[76] Eschmann KCJ, Bader R, Mecklinger A (2020) Improving episodic memory: Frontal-midline theta neurofeedback training increases source memory performance. Neuroimage 222, 117219.

[77] Enriquez-Geppert S, Huster RJ, Figge C, Herrmann CS (2014) Self-regulation of frontal-midline theta facilitates 
memory updating and mental set shifting. Front Behav Neu$\operatorname{rosci} \mathbf{8}, 420$

[78] Klimesch W, Doppelmayr M, Schimke H, Ripper B (1997) Theta synchronization and alpha desynchronization in a memory task. Psychophysiology 34, 169-176.

[79] Altan S, Berberoglu B, Canan S, Dane Ş (2016) Effects of neurofeedback therapy in healthy young subjects. Clin Invest Med 39, 27496.

[80] Garcia J, Kimeldorf DJ, Koelling RA (1955) Conditioned aversion to saccharin resulting from exposure to gamma radiation. Science 122, 157-158.
[81] Fouquet N, Oberling P, Sandner G (2001) Differential effect of free intake versus oral perfusion of sucrose in conditioned taste aversion in rats. Physiol Behav 74, 465-474.

[82] la Vaque TJ, Hammond CD, Trudeau D, Monastra V, Perry J, Lehrer P, Matheson D, Sherman R (2002) Template for developing guidelines for the evaluation of the clinical efficacy of psychophysiological interventions. Appl Psychophysiol Biofeedback 27, 273-281. 